\title{
Diacronie
}

Studi di Storia Contemporanea

$\mathrm{N}^{\circ} 20,4 \mid 2014$

II diritto militante

\section{Il delitto di Roncosaglia}

\section{Simeone Del Prete}

\section{(2) OpenEdition \\ Journals}

\section{Edizione digitale}

URL: http://journals.openedition.org/diacronie/1691

DOI: 10.4000/diacronie. 1691

ISSN: 2038-0925

\section{Editore}

Association culturelle Diacronie

\section{Notizia bibliografica digitale}

Simeone Del Prete, « II delitto di Roncosaglia », Diacronie [Online], № 20, 4 | 2014, documento 4, Messo online il 01 décembre 2014, consultato il 01 mai 2019. URL : http://journals.openedition.org/ diacronie/1691 ; DOI : 10.4000/diacronie.1691 


\title{
Diacronie
}

N. 20 | 4|2014 Il diritto militante

4/

\section{Il delitto di Roncosaglia}

\author{
Simeone DEL PRETE*
}

Il lavoro si propone di analizzare il ruolo e le caratteristiche dell'avvocatura militante del legale bolognese Leonida Casali, esponente di spicco sia del Partito Comunista Italiano, sia del Comitato di Solidarietà Democratica nel secondo dopoguerra. Attraverso lo studio di vari casi particolari, quali i processi contro ex partigiani e militanti politici, si è puntato a far emergere l'intrinseca politicità dell'operato dell'avvocato, mostrandone, da una parte l'autorevolezza e l'autonomia operativa, dall'altra lo stretto rapporto che lo legava al PCI e al CSD. Tali aspetti permettono di delineare la figura dell'avvocato Casali come un unicum nel panorama della giustizia italiana dell'immediato dopoguerra.

I

l cosiddetto delitto di Roncoscaglia rappresentò uno dei numerosi casi in cui l'avvocato Casali prestò la propria opera in difesa di un ex partigiano. Tale caso risulta però assai rilevante poiché ebbe un grandissimo clamore nell'opinione pubblica e nella lotta politica tra Dc e Pci, tanto da rappresentare quasi un unicum per intensità nel panorama del dopoguerra italiano. Di tale delitto, ossia dell'omicidio di un'anziana signora, furono incolpati sei ex combattenti partigiani, i quali, dal momento che non sussistevano prove concrete della loro colpevolezza, furono costretti dalla violenza delle forze di polizia a rendere confessioni nelle quali ammettevano di aver ordito e eseguito il delitto. Tale situazione, dunque, rappresentò una grande battaglia politica fatta di discreditamento e di propaganda, dalla quale Casali ed il suo assistito uscirono vincitori. 
La mattina del 25 gennaio 1948, la sedicenne Pia Pini, recatasi, come di consueto, presso la casa di Maria Zanarini, presso la quale prestava servizio come massaia, trovò la porta della casa aperta, vide una pozza di sangue vicino alla soglia ed, affacciatasi, scorse all'interno della cucina, disteso per terra il corpo senza vita della signora Zanarini. Spaventata, la giovane corse nella propria abitazione e riferì al padre Primitivo ed ai fratelli Alberto, Mauro e Remigio ciò che aveva visto. I tre si recarono immediatamente al comando dei carabinieri di Roncoscaglia per denunciare l'accaduto.

I carabinieri della vicina stazione di Sestola, venuti, grazie ad una telefonata anonima, a conoscenza di un cadavere rinvenuto nella frazione di Roncoscaglia, si recarono immediatamente sul luogo, dove poterono appurare che il cadavere presentava lesioni alla testa, al petto e alla gola apportate probabilmente attraverso un oggetto contundente, ipotizzando come dinamica dell'assassinio una colluttazione seguente ad un tentativo di rapina, dal momento che nella casa risultava evidente uno stato di soqquadro generale, anche se sul corpo della Zanarini non erano ravvisabili segni di lotta ${ }^{1}$.

Durante l'interrogatorio a Pia Pini, che intanto era stata trattenuta presso il comando di Roncoscaglia per ulteriori accertamenti, emerse il fatto che i tre fratelli della giovane erano rientrati in casa molto tardi la sera del delitto, approssimativamente intorno alle due del mattino e che quindi sicuramente non si trovavano in casa all'orario dell'omicidio, ipotizzato dagli inquirenti intorno alle nove, come in principio aveva asserito Pia. Poiché la voce pubblica, che descriveva i tre fratelli come esaltati ex combattenti, accusava del delitto i fratelli della Pini e poiché questa, interrogata circa il modo in cui i propri fratelli avessero trascorso la notte del 24 gennaio e circa l'ora del loro rientro in casa, aveva fornito dati incongruenti e contraddittori, i carabinieri ritennero di procedere alla perquisizione dell'abitazione della famiglia Pini. Durante la stessa vennero così sequestrati un paio di pantaloni di tela sui quali vennero notate delle macchie aventi caratteristiche simili a quelle di sangue, indumento che i componenti della famiglia Pini concordemente dichiararono appartenere al loro congiunto Alberto $^{2}$.

Dato che i sospetti già gravanti su costui e sui suoi fratelli Remigio e Mauro venivano rafforzati dal sequestro del suddetto indumento, i carabinieri procedettero al fermo dei tre indiziati, onde svolgere più accurate indagini dirette ad accertare la fondatezza o meno degli elementi di accusa a loro carico. Tutti e tre i fermati, interrogati si professavano estranei al delitto, fornendo particolareggiate indicazioni sul

${ }^{1}$ ISPER, Fondo Casali, Sez. 2, Sett. 2, b. 103, f. 25.

2 Ibidem. 
modo in cui avevano trascorso la sera del 24 gennaio, che trovavano conferma negli accertamenti eseguiti dagli inquirenti3 ${ }^{3}$

Intanto, l'Istituto di Medicina Legale dell'Università di Bologna, cui erano stati rimessi per i dovuti accertamenti tecnici i pantaloni sequestrati in casa Pini, in una nota del 17 febbraio 1948 informava che le macchie esistenti sull'indumento sequestrato dovevano «con tutta probabilità non essere riconducibili a sangue umano»4. Venuto così a mancare anche l'ultimo elemento di sospetto giustificante il fermo dei tre fratelli Pini, con provvedimento in data 26 febbraio 1948 del Pretore di Pavullo, veniva ordinata la loro scarcerazione. Così si concludeva la prima fase delle indagini di polizia.

Senonché i carabinieri della squadra giudiziaria del gruppo di Modena, in data 9 marzo 1949 nell'ambito di nuove indagini condotte nella zona di Montecreto e Sestola, dichiararono 5 di essere riusciti ad identificare i responsabili dell'omicidio Zanarini, riferendo che essi, interrogati, avevano finito con il confessare la loro partecipazione all'omicidio, fornendo ampi particolari in ordine all'organizzazione ed all'esecuzione del delitto. In base a tale denuncia, il procuratore della Repubblica di Modena procedette contro sei ex partigiani, tra i quali spiccavano i nomi di due dei fratelli Pini, Mauro e Alberto. Oltre ai due fratelli Pini furono arrestati anche Gino Bonaccorsi, Silvio Fiocchi, Mario Covili, Francesco Santini e Eraldo Querciagrossa, tutti ex combattenti in formazioni partigiane legate al Partito Comunista Italiano. Solo l'ultimo di questi fu difeso in giudizio dall'avvocato Casali, che però, come si vedrà in seguito, riuscì a dimostrare la mendacità delle presunte ammissioni di colpa, riuscendo a scagionare tutti gli imputati.

Stando al rapporto dei carabinierí, infatti, tutti gli indagati si erano resi rei confessi ammettendo di aver ordito e organizzato l'omicidio a scopo di rapina a danno della signora Zanarini; in particolare secondo le deposizioni attribuite a Pini Mauro la banda, scorgendo la possibilità di arricchirsi procurandosi illegalmente il denaro della danarosa signora, ne avrebbero orchestrata e realizzata l'uccisione. Nella stessa deposizione però, stando sempre al rapporto dei carabinieri, lo stesso avrebbe confessato di aver agito più per motivi ideologici che per scopo di rapina, dato che la danarosa signora Zanarini era la padrona della sorella e che tale atto avesse come movente la lotta di classe.

\footnotetext{
3 Ibidem.

4 Nota dell'Istituto di Medicina Legale dell'Università di Bologna. ISPER, Fondo Casali, Sez.2, Sett. 2, b.103, f. 25 .

5 Rapporto della Squadra giudiziaria dei carabinieri di Modena. ISPER, Fondo Casali, Sez.2, Sett. 2, b. 103 , f. 25 .

${ }^{6}$ Ibidem.
} 
Tali confessioni, secondo le dichiarazioni successivamente rilasciate dagli indagati, però, furono estorte con la violenza da parte dei carabinieri che le ottennero soltanto torturando e seviziando ripetutamente gli imputati con calci, pugni, manganellate e attraverso la privazione del sonno. Gli imputati dichiararono inoltre di essere stati legati ad un tavolinetto e poi fatti oggetto di bastonate e scudisciate, oltre che di avere subito umiliazioni quali l'essere stati costretti al bere urina7.

Infatti, nel corso degli interrogatori successivi all'arresto ${ }^{8}$, Mauro Pini smentì categoricamente quanto dichiarato in precedenza e rivelò di aver sottoscritto un verbale già redatto dai carabinieri a causa delle minacce e delle percosse infertegli dagli stessi ufficiali, asserendo la sua completa estraneità al delitto. Tale estraneità fu inoltre corroborata da un alibi che fu comprovato: egli dichiarò di essersi trattenuto fino alle 23 a casa sua, per poi trasferirsi a casa di un amico dove avrebbe trascorso le ore mancanti al suo rientro a casa. Anche gli altri cinque negarono qualsiasi partecipazione al fatto, dichiarando di essere stati costretti a firmare le dichiarazione ed ammettere così la loro partecipazione al delitto sotto la violenza e la tortura usata nei loro confronti da parte dei carabinieri investigatori 9 .

Interrogati lungamente $\mathrm{i}$ verbalizzanti, questi smentirono categoricamente e recisamente ciò che gli imputati avevano affermato nei loro interrogatori e cioè di essere stati da questi sottoposti a misure di rigore, violenze e minacce per confessarsi autori dell'omicidio Zanarini. Essi precisavano che le singole dichiarazioni nei loro particolari erano state loro fornite dagli stessi imputati dopo vari interrogatori, sulla scorta delle notizie che avevano raccolto tra la popolazione e non attraverso minacce o percosse.

Onde accertare se le violenze che gli imputati dichiaravano di aver subito ad opera dei verbalizzanti trovassero riscontro con dati obiettivi su di essi evidenziabili, venne disposta una perizia medica ${ }^{10}$. Nella stessa, sebbene su qualcuno degli imputati fossero stati dal perito rilevati cicatrici ed ematomi, non fu possibile avverarne le cause e l'epoca di produzione, pur non escludendo che potessero avere relazioni con i fatti lamentati dai prevenuti.

A completamento di tale laboriosa e complessa istruttoria venivano escussi una seconda volta i verbalizzanti i quali negarono di aver adoperato metodi violenti, confermando che gli indagati avevano senza alcuna forma coercitiva reso le confessioni risultanti dagli interrogatori che avevano sottoscritto.

7 ISPER, Fondo Casali, Sez. 2, Sett. 2, b. 103, f. 25.

8 Ibidem.

9 Ibidem.

10 Ibidem. 
Come esplicitato dal Pubblico Ministero nella requisitoria,

dall'esame delle risultanze istruttorie dovette riconoscersi che gli elementi accusatori da questa emersi a carico dei prevenuti, sostanziandosi quasi unicamente, sulle confessioni di colpevolezza, non apparivano sufficienti a legittimare l'esperimento del giudizio, dato che le confessioni che le integrano non solo vennero, in definitiva, ritrattate da tutti gli imputati, ma i fatti che ne formarono oggetto non trovarono riscontro in alcuna emergenza dell'istruttoria che ne potesse convalidare l'attendibilità ${ }^{11}$.

Nonostante ciò, la sentenza di primo grado, redatta dal procuratore Baratti, risultava di segno opposto: secondo il procuratore infatti, le confessioni costituivano prove sufficienti a carico di tutti i prevenuti per il loro rinvio al giudizio della Corte d'Assise.

Nella sentenza di primo grado, infatti, si adduceva la tesi secondo cui costoro avrebbero

cercato di togliere valore alle ampie e particolareggiate confessioni rese che avevano trovato riscontro nelle obiettive risultanze dei fatti con il solito pretesto che esse fossero state estorte con violenza, che nemmeno nella specie sarebbero state limitate a semplici percosse ma si sarebbero concretate in vere e proprie feroci torture, che si ritengono poco plausibili. Anche a voler trascurare la versione negativa opposta dai pretesi seviziatori, l'asserzione degli imputati contrastava anzitutto con la mancanza assoluta di dati obiettivi ricercati non appena l'assenza delle sevizie fu mossa perché il medico delle carceri rilevò lievissime ecchimosi delle quali persino gli stessi indagati si erano disinteressati ${ }^{12}$.

Proprio dopo tale sentenza e in preparazione del passaggio in Corte d'Assise, è verosimilmente presumibile che il Partito Comunista abbia incaricato l'avvocato Casali di difendere uno dei sei imputati, Eraldo Querciagrossa, il quale risultava iscritto e militante del partito.

La molto meticolosa difesa dell'avvocato bolognese fu incentrata sulle clamorose incongruenze che emergevano tra le dichiarazioni estorte con la violenza e la verosimiglianza delle stesse e su elementi che ne inficiavano pesantemente la spontaneità. Alla evidente carenza di naturalezza, testimoniata da una mole di particolari che difficilmente potevano essere stati confessati da un colpevole, infatti, si 
aggiungeva anche un difetto di verosimiglianza di alcuni particolari dei fatti confessati; innanzitutto le narrazioni che i prevenuti avrebbero fatto dell'organizzazione e dell'esecuzione del delitto non risultavano verosimili soprattutto laddove si evidenziava la necessità di correi per attuare il proposito criminoso in danno di una persona anziana che viveva in una casa del tutto isolata. Non è irrilevante per dimostrare l'inverosimiglianza della confessione in esame porre, come fece Leonida Casali, in evidenza l'esagerata sproporzione dell'armamento in possesso dei partecipanti al delitto in rapporto alla limitatissima capacità difensiva della vittima designata ${ }^{13}$. Ma ciò che più inficia la verosimiglianza delle confessioni è la versione circa la divisione del bottino della rapina tra i compartecipi del delitto, che risultava fortemente sproporzionata, tanto che ad uno dei sei, secondo le confessioni, non sarebbe stata assegnata alcuna parte del bottino ${ }^{14}$.

Proprio grazie allo scrupoloso lavoro di analisi delle confessioni e di confronto tra le stesse e le prove giudiziali, Casali riuscì ad ottenere il proscioglimento per insufficienza di prove di tutti i sei imputati e la loro immediata scarcerazione. La bontà e il successo di tale lavoro sono ravvisabili anche e soprattutto nella sentenza della Corte d'Appello:

Vero è che di qualsiasi confessione, giudiziale o stragiudiziale, il giudice può trarre in materia penale elementi per il suo libero convincimento; ma è altresì vero che in ogni caso essa, per acquistare rilevanza probatoria, anche solo indiziaria oltre a non essere invalidata da altri elementi di prova, deve rispondere a quei requisiti di spontaneità, verosimiglianza, determinatezza, precisione ed esplicitezza che di norma ne caratterizzano l'attendibilità, anche se poi ritrattata. A tali requisiti di certo non rispondevano le confessioni in esame: in ordine a queste è anzi da osservare che la loro attendibilità, se non del tutto annullata fu certamente di molto affievolita dalle prove documentali che confermavano gli alibi dedotti dai prevenuti in base ai quali, se non con assoluta certezza, almeno con plausibile probabilità e verosimiglianza, è lecito ritenere che non ci siano prove che la sera del 24 gennaio del 1948 gli imputati fossero presenti a Roncoscaglia alla consumazione dell'omicidio della Zanarini'5.

\footnotetext{
${ }_{13}$ Secondo le confessioni dei sei, infatti, gli stessi sarebbero stati dotati di circa una ventina di armi contundenti, tra mazze, bastoni e martelli. Tale numero, probabilmente gonfiato dai membri delle forze dell'ordine proprio per farlo apparire come una prova inconfutabile dell'intenzionalità dell'omicidio, appariva del tutto improbabile, dal momento che ognuno degli indagati avrebbe dovuto sorreggere tre corpi contundenti.

${ }^{14}$ Le confessioni estorte con la violenza metterebbero in luce una divisione del bottino del tutto inusuale, dal momento che, secondo le medesime, a Silvio Fiocchi non sarebbe spettato nulla del bottino, disvelando in maniera evidente, se non l'innocenza dello stesso, almeno l'erroneità del movente della rapina.

15 Ibidem.
} 


\section{L'avvocato Casali tra difesa militante e lotta politica}

Il caso appena preso in esame, relativo alle vicende giudiziarie scaturite dall'omicidio di Maria Zanarini, può considerarsi di interessante rilevanza politica non solo poiché riguardante false accuse mosse nell'immediato dopoguerra ad un gruppo di ex partigiani da parte di carabinieri e magistrati tra loro conniventi, ma anche per il grande risalto che tali vicende ebbero nel dibattito politico contemporaneo esterno ma soprattutto interno al Partito Comunista Italiano. Se da una parte, infatti, tali vicende possono essere ascritte a un novero assai ampio di processi fondati su accuse senza fondamento rivolte a ex partigiani nei primi anni seguenti il secondo conflitto mondiale, dall'altra è innegabile che tale questione abbia rappresentato uno dei perni della strategia denigratoria attuata dal governo democristiano di De Gasperi e di Scelba nei confronti del PCI e della stessa lotta partigiana. Tale strategia trovava facile sponda in una magistratura e in un corpo di polizia, che non avendo subito adeguati processi di epurazione e di rinnovamento, annoveravano tra le loro fila membri che non solo si erano compromessi con il regime fascista, ma che addirittura potevano considerarsi in continuità con lo stesso, essendosi formati ed avendo raggiunto posizioni di vertice proprio nel corso del ventennio fascista ${ }^{16}$.

Non vi è dubbio, infatti, che tale caso, al di là del fatto di cronaca in sé, fosse non solo un chiaro esempio delle tensioni sociali e politiche che, scaturite durante il fascismo, risultavano nell'immediato dopoguerra tutt'altro che sopite, ma anche un premeditato e freddamente calcolato tentativo denigrazione giuridico-politica nei confronti del movimento partigiano ed in particolare della sua frangia più organizzata, quella comunista. L'obiettivo di tale strategia era quello di criminalizzare i comunisti attraverso l'omologazione della conflittualità sociale con la delinquenza comune, mostrandoli all'opinione pubblica, anche grazie allo smodato interesse dei giornali moderati a tali vicende, come delinquenti e agitati intenti a rompere il fragile equilibrio su cui si sorreggeva la neonata Repubblica.

In tale caso e, per diretta conseguenza, in tale battaglia, Leonida Casali rivestì un ruolo di primo ordine: oltre ad essere il difensore dell'ex partigiano Eraldo Querciagrossa, egli fu uno dei veri e propri imbastitori del dibattimento politico sulla questione. L'avvocato infatti intrattenne un'articolata e proficua corrispondenza

16 NEPPI MODONA, Guido, La magistratura dalla liberazione agli anni cinquanta, in BARBAGALLO, Francesco, op. cit., pp. 84-85. 
relativa al caso con Mario Ricci ${ }^{17}$ e con Umberto Terracini, entrambi parlamentari ed esponenti di rilievo sia del Partito Comunista Italiano, sia del Comitato di Solidarietà Democratica, organo atto a difendere le libertà democratiche e a fornire assistenza legale e sostegno materiale agli arrestati per motivi politici e alle loro famiglie, il cui impulso costitutivo, scaturito nella fondazione del comitato dell'agosto del 1948, è da attribuire allo stesso on. Terracini. Ciò che emerge da tale carteggio ${ }^{18}$, che in seguito sarà analizzato in maniera approfondita, è quella della solida politicità della figura dell'avvocato bolognese: egli non fu soltanto un avvocato di fiducia per la dirigenza locale e nazionale del Pci, ma un autentico compagno in grado di elaborare analisi politiche e strategiche e di influenzare con la sua autorevolezza lo svolgimento di una campagna politica.

L'avvocato Casali, dunque, non solo si prese in carico la difesa di uno degli ex partigiani imputati, riuscendo a scagionare tutti gli imputati dalle accuse, ma rivestì anche un ruolo fondamentale nell'approccio politico al caso: il disvelare l'artificiosità delle accuse rivolte agli ex partigiani poteva infatti rappresentare un'ulteriore conferma della strategia anticomunista del partito al potere e di conseguenza un'occasione di propaganda politica a favore del Pci. E' ravvisabile in tale atteggiamento quella che da più parti è stata definitiva la linea del “doppio binario" adoperata dalla difesa: da una parte la difesa militante di Casali fu per ovvi motivi difensiva, dall'altra essa fu offensiva poiché atta a contestare una situazione di malcostume e di repressione nei confronti di ex partigiani che non faceva altro che avvantaggiare il moderatismo della Democrazia Cristiana. ${ }^{19}$ Significativo appare quindi rilevare come lo sforzo portato avanti da Casali si muovesse da una parte difensivamente per assistere nelle cause gli imputati, dall'altra offensivamente denunciando la tendenziosità dell'azione persecutoria nei confronti degli ex combattenti partigiani, il cui obiettivo non era l'assoluzione degli

\footnotetext{
17 Nell'unica lettera pervenutaci dell'on. Ricci all'avvocato Casali risulta interessante notare come il primo si fosse firmato come "Comandante Armando", pseudonimo che lo stesso utilizzava durante le azioni della divisione partigiana di cui era al comando e a cui peraltro aveva dato il nome, la Divisione Modena-Armando, passata alla storia per la fondazione della prima Repubblica Partigiana in Italia, la Repubblica di Montefiorino. L'ipotesi che i due avessero avuto contatti durante l'esperienza resistenziale è avvalorato solo flebilmente dal fatto che i due operassero in territori contigui; si ritiene un dato importante da rilevare il fatto che il primo specifichi il proprio pseudonimo al secondo poiché anche egli ex partigiano.

${ }_{18}$ ISPER, Fondo Casali, Sez. 2, Sett. 2, b. 103, f. 25.

19 Per un'analisi del diritto come strumento offensivo e difensivo si rimanda al lavoro svolto da Liora Israel sul mondo del diritto e delle avvocature militanti soprattutto in Francia e specialmente al volume Le armi del diritto (Milano, Giuffré, 2012) nel quale la studiosa ravvede nel diritto sia un carattere offensivo, atto a far valere e a conquistare diritti, sia un carattere difensivo, poiché imposto da una indagine o da una imputazione.
} 
imputati come scopo in sé compiuto, bensì come affermazione dei valori costituzionali nati proprio grazie alla lotta dei partigiani durante la Resistenza.

L'assoluzione degli imputati, dunque, oltre che all'avere salvato da un'ingiusta pena degli innocenti, fu funzionale anche al disvelare come la magistratura del tempo fosse legata a doppio filo al governo targato Dc e di come la comunanza di intenti dei due organi, ossia quella di incolpare comunisti innocenti e di farli passare nell'opinione pubblica come criminali assetati di sangue, fosse atta a screditare il Pci e la Resistenza stessa e a creare dissenso nei confronti di un partito e di un movimento che, con il suo radicamento territoriale, specialmente in Emilia Romagna, e con la radicalità che lo contraddistingueva, poteva insidiare il potere democristiano e l'intero assetto geopolitico dell'Europa occidentale.

Per una ricostruzione storica di quale siano stati il ruolo e la rilevanza politica della difesa militante svolta da Leonida Casali nel caso dell'omicidio di Montecreto si è ritenuto necessario riportare ed analizzare il carteggio relativo al caso che l'avvocato intrattenne con l'on. Terracini, che era evidentemente interessato allo stesso per la rilevanza politica di cui si è scritto pocanzi, tant'è che fece pervenire a Casali attraverso l'allora sindaco di Bologna nonché compagno di partito e amico personale del legale, Giuseppe Dozza, una richiesta di visione del materiale relativo al caso. La prima di queste lettere, inviata dall'avvocato al senatore il 4 novembre 1950 riporta quanto segue:

\section{Caro Terracini,}

Dozza mi ha comunicato di spedire a te il materiale relativo al processo contro Querciagrossa ed altri sei, imputati di omicidio a scopo di rapina che sono stati assolti dalla Corte di Assise di Modena con la sentenza del 20 ottobre scorso per non avere commesso il fatto. Detti imputati, sei dei quali partigiani, avevano in sede di polizia rese delle confessioni lunghissime e particolareggiate. [...] Tutti gli imputati invece erano completamente estranei al delitto. E' stata una ignobile e infame montatura di alcuni carabinieri, con l'appoggio e la connivenza di alcuni loro superiori e, con molta probabilità, del giudice istruttore. [...] Per fortuna ho potuto dimostrare l'innocenza del mio difeso e conseguentemente degli altri, [...] ponendo in rilievo le insanabili contraddizioni della polizia giudiziaria che partendo da presupposti dimostratisi poi erronei, aveva posto in essere una costruzione del tutto inverosimile e fantastica. Comunque è certo che le confessioni furono unicamente il prodotto della violenza morale e fisica subita dagli imputati e ciò, questa volta, è risultato in modo inoppugnabile. Purtroppo grave responsabilità in ordine a tali fatti, che oramai sono diventati un metodo, risale anche all'autorità 
giudiziaria inquirente e requirente perché, pur essendo a conoscenza dei continui abusi e reati che si commettono da parte della polizia giudiziaria, li copre e li favorisce. [...] $\gg^{20}$.

Tale epistola, una copia conforme della quale fu conservata da Casali nel suo archivio, dimostra innanzitutto una familiarità innegabile tra il mittente ed il ricevente della stessa; infatti, nonostante la lettera fosse indirizzata all'ufficio Senatoriale di Terracini e di conseguenza rappresentasse parte di una corrispondenza ufficiale, il tono risulta tutt'altro che formale; Casali dava del tu al Senatore e non risparmiava giudizi di merito sull'operato delle forze dell'ordine e della magistratura. Tale atteggiamento dimostra in maniera inoppugnabile quanto a Casali fosse riservata una collocazione interna all'organismo dirigenziale del Partito Comunista Italiano tutt'altro che subalterna, cosa che ci si potrebbe aspettare per un avvocato che, seppur godendo della fiducia dell'apparato comunista, non fosse interno alla sua logica. Ciò, ovviamente, non vale per Leonida Casali che, con la sua militanza pluriennale, rappresentava molto di più che un avvocato di cui fidarsi. Egli, infatti, può essere definito sia avvocato del partito, sia avvocato nel partito, in quanto non solo rispettava di buon grado le direttive che gli arrivavano dagli organi dirigenti del Pci, ma aveva voce in capitolo nel delineare le stesse, se non addirittura nell'influenzare la linea politica del partito.

Tale peculiarità risulta assai più evidente nella risposta che il Senatore diede a Casali nella lettera dell'8 novembre 1950, in cui viene messa in luce tutta la preponderanza del ruolo di questo ultimo. Proprio per questo si è ritenuto di utilità storica riportare in seguito una parte della stesa.

Caro compagno avvocato,

[...] Mi riprometto di esaminarli attentamente ${ }^{21}$ perché mai ci è stata data occasione migliore per denunciare clamorosamente l'ignominia dei procedimenti giudiziari abbandonati alla polizia e dei quali la magistratura non fa che ratificare a posteriori le conclusioni, salvo poi che, come nel caso attuale, essa sbatta contro una evidenza validamente sostenuta da agguerriti difensori. Non so tuttavia se non sia meglio che io attenda la sentenza, prima di impostare la battaglia politica. Ti prego di esprimermi il tuo avviso ${ }^{22}$.

${ }^{20}$ ISPER, Fondo Casali, Sez.2, Sett.2, b.103, f.25.

${ }^{21}$ L'on. Terracini si riferisce ai materiali relativi al caso inviatigli da Casali a seguito della lettera pocanzi citata.

${ }^{22}$ Ibidem. 
Quale sia stata l'indicazione data da Casali a Terracini non è dato di sapere, dal momento che non ci è pervenuta alcuna lettera di risposta alla precedente, ma è ipotizzabile che il responso del primo sia stato appunto quello di ritardare l'imbastimento della battaglia politica a dopo la sentenza, dal momento che il carteggio $^{23}$, principalmente incentrato sui trasferimenti dei fascicoli del caso riprenderà solo nel 1952 e che le interrogazioni parlamentari presentate dagli onorevoli Terracini e Ricci sono anche esse successive a tale anno. Sempre al 1952 risale anche il processo intentato per vilipendio contro l'organo di stampa «Vie Nuove», rivista legata al PCI e fondata da Luigi Longo nel 1946, a causa della pubblicazione di una vignetta satirica svolgente appunto il tema delle confessioni estorte con la violenza dalla polizia nella fase istruttoria dei processi ${ }^{24}$.

Le ultime righe di tale epistola, con la richiesta da parte di un Senatore della Repubblica a Casali di un consiglio su quale fosse il momento più opportuno per imbastire una battaglia politica, dimostrano ancora una volta quanto il ruolo dell'avvocato non fosse esclusivamente quello di un consulente giuridico, ma anzi avesse ricadute profonde addirittura sulle scelte politiche del gruppo parlamentare del Pci. Su tale ambivalenza dunque si gioca il ruolo dell'avvocatura militante di Casali: da una parte il partito dispone di lui come di un fedele e fidato servitore, dall'altra la sua esperienza da forense, combinata con un impegno politico di primo ordine, che lo portò a rivestire incarichi politici a livello locale, lo rende un fine conoscitore degli assetti statali e della strategia politica.

La sua adesione al Pci, dunque, appare totale sia a livello ideologico, sia a livello strategico; lo specchio di tale adesione, però, è una rilevanza e un'autorevolezza a lui riconosciuta dalle alte sfere del Partito Comunista Italiano, cosa che colloca l'avvocato Casali in un ruolo che, se non del tutto inedito, fu ben poche volte notato nella storia delle avvocature militanti in Italia. Nella sua persona, dunque, la figura dell'avvocato militante si mescola a quella dell'uomo di partito, mostrandoci la complessità del ruolo di un uomo che ha inequivocabilmente segnato la storia del dopoguerra italiano.

\footnotetext{
23 Ibidem.

24 Proprio in occasione di tale processo riprenderà il carteggio tra Casali e Terracini. Tale carteggio, principalmente incentrato sullo scambio di un fascicolo che Terracini curiosamente non riusciva a trovare nel disordine del suo ufficio, sarà l'occasione per l'onorevole genovese di invitare l'avvocato a presenziare, in qualità di consulente, come dirà lo stesso in una lettera del 29 aprile 1952, «a quel processo in quel tribunale».
} 


\section{* L'autore}

Simeone Del Prete ha conseguito la Laurea triennale in Storia presso l'Università di Bologna con una tesi di laurea in storia economica sul conflitto nordirlandese, frutto di ricerche effettuate presso l'University College Cork. Attualmente frequenta il corso di Laurea Magistrale in Scienze Storiche presso il medesimo ateneo.

URL: < http://www.studistorici.com/progett/autori/\#DelPrete >

\section{Per citare questo articolo:}

DEL PRETE, Simeone, «ll delitto di Roncosaglia», Diacronie. Studi di Storia Contemporanea : II diritto militante, 29/12/2014,

URL:< http://www.studistorici.com/2014/12/29/delprete_numero_20/ >

Diacronie Studi di Storia Contemporanea 3 www.diacronie.it

Risorsa digitale indipendente a carattere storiografico. Uscita trimestrale. redazione.diacronie@hotmail.it

Comitato di redazione: Jacopo Bassi - Luca Bufarale - Elisa Grandi - Deborah Paci - Fausto Pietrancosta - Matteo Tomasoni - Luca Zuccolo

Diritti: gli articoli di Diacronie. Studi di Storia Contemporanea sono pubblicati sotto licenza Creative Commons 2.5 . Possono essere riprodotti a patto di non modificarne i contenuti e di non usarli per fini commerciali. La citazione di estratti è comunque sempre autorizzata, nei limiti previsti dalla legge. 